\title{
PRESENCIA DE Cryptococcus neoformans EN EXCRETAS DE PALOMAS URBANAS EN SAN MIGUEL DE TUCUMÁN - ARGENTINA
}

\author{
(Presence of Cryptococcus neoformans in urban pigeons excreta from \\ San Miguel de Tucumán - Argentina)
}

\author{
Alvarez $\mathbf{C}^{\mathbf{1}}$., Salim $\mathbf{R}^{2}$., Runco $\mathbf{R}^{1,2}$ \\ 1- Laboratorio de Micología del Hospital del Niño Jesús. \\ Pasaje Hungría 750. (4000) Tucumán. R. Argentina. \\ 2- Cátedra de Micología. Instituto de Microbiología \\ Facultad de Bioquímica, Química y Farmacia. Universidad \\ Nacional de Tucumán. Ayacucho 491- (4000) Tucumán. R. Argentina \\ bqco_christianalvarez@hotmail.com
}

Palabras claves: Cryptococcus neoformans, excretas de palomas, espacios públicos, antifúngicos.

Key words: Cryptococcus neoformans, pigeon's excreta, public spaces, antifungal.

RESUMEN

Con la finalidad de detectar la presencia del complejo Cryptococcus neoformans en excretas de palomas (Columba livia) que habitan los espacios públicos del perímetro urbano de San Miguel de Tucumán, Argentina, se recogieron 100 muestras de 5 localizaciones diferentes. Como medio de aislamiento se empleó Agar-Semillas de Niger y las levaduras fueron identificadas mediante la presencia de cápsula, prueba de la ureasa, producción de fenol-oxidasa, asimilación de carbohidratos y crecimiento a $37^{\circ} \mathrm{C}$. La especie fue determinada usando el medio Canavanina-Glicina-Azul de bromotimol.

C. neoformans var. neoformans fue aislado en todas las 55 muestras de las áreas estudiadas, reflejando una amplia distribución en la zona céntrica de la ciudad preferentemente en excretas secas acumuladas. La sensibilidad in vitro frente a Fluconazol, Itraconazol, Anfotericina B y Voriconazol fue determinada por el método de difusión en placa usando tabletas NeoSensitabs. Todos los aislamientos fueron sensibles a los antifúngicos testeados.

\section{INTRODUCCIÓN}

Las enfermedades infecciosas emergentes son aquellas que reaparecen en una población o rango geográfico. Una vía para el entendimiento y prevención

Recibido el 27 de Marzo 2010

Aceptado el 2 de Julio 2010

\section{ABSTRACT}

With the purpose of detecting the presence of Cryptococcus neoformans complex in excreta of pigeons inhabitating public spaces belonging to the urban perimeter of San Miguel de Tucumán, Argentina, one hundred samples were collected from five different locations. Niger seed agar plates were used as means of isolation and yeasts were identified through the presence of capsule, urease test, fenoloxidase production, carbohydrate assimilation andgrowth at $37^{\circ} \mathrm{C}$. Species was determined with the CGB method.

C. neoformans var. neoformans was isolated in all 55 samples of the studied areas showing a wide distribution downtown, mainly in dried excreta settlings. In vitro sensibility in front of Fluconazol, Itraconazol, Anfotericina $B$ and Voriconazol was fixed by means of the diffusion method by using Neo-Sensitabs tablet. All isolations proved to be sensible to the tested antifungal.

de estas enfermedades, es definir los factores predisponentes que pueden incluir: cambios ecológicos, alteraciones demográficas, viajes y comercio, tecnología, adaptación de los microorganismos y medidas de salud pública. (Taubenberger \& Morens, 2006). La diseminación geográfica de un microorganismo infeccioso puede ocasionar un brote, como el de criptococosis producido por $\boldsymbol{C}$. gattii en la Isla de Vancouver, Canadá (Hoang et al., 2004).

La criptococosis es una micosis sistémica de curso subagudo o crónico y de amplia distribución mundial. El 
agente etiológico es una levadura capsulada, del género Cryptococcus, capaz de causar enfermedades serias y aún fatales en individuos inmunocomprometidos, especialmente aquellos con SIDA (Mattsson et al., 1999).

El hombre puede infectarse por la inhalación de propágulos aéreos de Cryptococcus a partir de fuentes ambientales (Ellis \& Pfeiffer, 1990; Casadevall \& Perfect, 1998; Heitman \& Lin, 2006).

C.neoformans y $\boldsymbol{C}$. gattii, son las especies patógenas más importantes del denominado complejo Cryptococcus neoformans. Estas levaduras presentan, entre otras, importantes diferencias en su ecología, epidemiología de la micosis que producen, y corresponden a grupos monofiléticos y divergentes entre sí. (KwonChung et al., 2002, 2006; Heitman \& Lin, 2006). Diversas investigaciones permitieron conocer los nichos ecológicos del complejo $\boldsymbol{C}$. neoformans, siendo aislado de heces de aves, de animales domésticos y salvajes, suelos y vegetales (Pal, 1984; Kielstein et al., 2000a, 2000b; Lazera et al., 1996; Granados et al., 2005; Tampieri, 2006; Heitman \& Lin, 2006; Rosario et al., 2005, 2008).

La paloma urbana (Columba livia) es, sin duda, la más importante como reservorio de la levadura, pero el estudio de una gran diversidad de especies de pájaros, deja claro que no es la única portadora de criptococos patógenos. En 1955, Emmons fue el primero en establecer la relación existente entre la levadura y las heces de estas aves. Estudios posteriores realizados por el propio Emmons $(1960,1995)$ y por otros investigadores de casi todo el mundo, han demostrado que las deposiciones de paloma son un importante reservorio de $\boldsymbol{C}$. neoformans.

La densidad poblacional de esta levadura en $C$. livia, es significativamente superior a la de otras fuentes, lo que sugiere que las excretas ofrecen condiciones sustentables, donde, posiblemente otros microorganismos no representen una competencia para este hongo (AbouGabal \& Atia, 1978; Staib \& Blisse, 1982). La falta de evidencias de una transmisión directa individuo-individuo, sustenta la hipótesis de que la infección podría adquirirse a partir de estas fuentes medioambientales por inhalación (Casadevall \& Perfect, 1998; Pollock, 2003).

Por otro lado, existen evidencias importantes que establecen una relación entre la distribución mundial de C. neoformans y los suelos contaminados con excretas de aves (Hsu et al., 1994; Emmons, 1995; López-Martínez \& Castañón-Olivares, 1995; Passoni, 1999; Chee \& Lee, 2005; Curo et al., 2005; González Acuña et al., 2007). Esta peculiar distribución geográfica hace importante el estudio de su nicho ecológico, como posible fuente de contagio para individuos susceptibles (Staib, 1987).

La sospecha de que estas aves sean la fuente de la enfermedad está comenzando a ser demostrada por la aplicación de métodos moleculares de tipificación que permiten comparar, con un alto grado de discriminación, las cepas encontradas en pacientes y en los animales desde su entorno más próximo (Heitman \& Lin, 2006; Rosario et al., 2008).

En un estudio epidemiológico sobre criptococosis en San Pedro, Provincia de Buenos Aires, Argentina, Bava y Negroni (1986) aislaron $\boldsymbol{C}$. neoformans en heces de palomas recogidas de palomares. En 1989, Rubinstein et al., aislaron C.neoformans del suelo de dos áreas endémicas de la provincia de Córdoba. En Santa Fe, Vanni et al., (1998), detectaron $\boldsymbol{C}$. neoformans en excretas de Columba livia principalmente de la zona céntrica y también en la zona costera. Pero, hasta donde sabemos, en la Argentina no hay estudios sobre la ocurrencia e identificación de esta levadura en excrementos de palomas recogidos en áreas públicas de Tucumán- Argentina, ni estudios sobre su sensibilidad antifúngica in vitro.

En 2008, iniciamos la investigación de la presencia de $\boldsymbol{C}$. neoformans en material recolectado de la madera en descomposición presente en cavidades de troncos de diferentes géneros y especies de árboles vivos, autóctonos de nuestra provincia, encontrados normalmente en los espacios públicos. Se detectaron altas concentraciones de propágulos del complejo C. neoformans en Jacaranda mimosifolia (nombre común Tarco) y Enterolobium contortisiliquum (Pacará) (Álvarez et al.,2009).

Por lo expuesto, se investigó la presencia de $\boldsymbol{C}$. neoformans en excretas de palomas desde espacios públicos del perímetro urbano de San Miguel de Tucumán, Argentina, para conocer su distribución ecológica local, y determinar la sensibilidad antifúngica in vitro de las cepas aisladas.

\section{MATERIALES Y MÉTODOS}

Esta investigación se realizó entre los meses de Febrero y Mayo de 2009, en el laboratorio de Micología del Hospital del Niño Jesús, Tucumán- R. Argentina.

Se recolectaron un total de 100 muestras del radio urbano de San Miguel de Tucumán, a partir de las heces desecadas de palomas presentes en los suelos de los siguientes espacios públicos: Plaza Bernardino Rivadavia, Plaza Hipólito Irigoyen, Plaza Independencia, Plaza General Justo José de Urquiza y Parque Avellaneda. En cada lugar se recogieron 20 muestras (Fig. 1).

Para la toma de muestras de las deyecciones secas de las aves (al abrigo del sol a fin de reducir la exposición a la radiación UV), se emplearon hisopos de algodón estériles, humedecidos en solución salina estéril adicionada de Cloranfenicol 0,2 g.L.

Cada muestra fue inoculada en placas individuales de Agar Semillas de Niger (NSA). Las placas fueron incubadas a temperatura ambiente al abrigo de la luz, con observación 
diaria y hasta 4 días.

Las colonias de color marrón oscuro, características de la especie, que permitió su diferenciación de los microorganismos acompañantes, fueron traspasadas a nuevas placas de NSA para la obtención de cultivos puros. Se realizaron además subcultivos en Sabouraud glucosa agar (SGA) para la realización de las pruebas de identificación morfológica y bioquímica.

La caracterización morfológica de los cultivos, se realizó mediante análisis macro-micromorfológicos de las colonias aisladas en NSA y SGA a $t^{\circ}$ ambiente.

La identificación bioquímica se realizó mediante estudios de fermentación de carbohidratos, asimilación de fuentes de carbono y nitrógeno realizadas por el método comercial API ${ }^{\circledR} 20$ C Aux (bioMériuex- France), detección de ureasa y fenoloxidasa, sensibilidad a cicloheximida a $25^{\circ} \mathrm{C}$ y $37^{\circ} \mathrm{C}$ y termotolerancia a $37^{\circ} \mathrm{C}$. La especie se determinó utilizando el medio agarizado de canavanina-glicina-azul de bromotimol (CGB) a $28^{\circ} \mathrm{C}$ durante cinco días.

C. neoformans fue identificado sobre la base de los siguientes caracteres positivos o negativos: ureasa, inositol $\mathrm{KNO} 3$, lactosa, maltosa, sacarosa, galactitol, pseudomicelio y modificación del color del CGB.

A partir de cada una de las muestras positivas, se evaluó su sensibilidad antifúngica por el método de difusión en placas de Agar Mueller-Hinton suplementado con 2\% de Glucosa y $0.5 \mathrm{ug} / \mathrm{ml}$ de azul de metileno (MHM). El inoculo se obtuvo a partir del cultivo puro en SGA de $48 \mathrm{hs}$, ajustándolo a $1-5 \times 10^{6} \mathrm{UFC} / \mathrm{mL}$ (equivalente al 0.5 de la escala de McFarland), en solución 0,15 M de cloruro de sodio estéril. Posteriormente, se embebió un hisopo estéril con el inoculo y se esparció sobre la superficie de la placa de Petri en tres direcciones. Las placas inoculadas se colocaron en estufa a $30^{\circ} \mathrm{C}$ durante 15 minutos. Al cabo de este tiempo, con la ayuda de una pinza estéril, se colocaron las tabletas Neo-Sensitabs (Rosco Diagnóstica) de: Anfotericina B [10 $\mu \mathrm{g}]$, Fluconazol [25 $\mu \mathrm{g}$ ], Itraconazol [8 $\mu \mathrm{g}$ ], y Voriconazol [1 $\mu \mathrm{g}$ ] por placa e incubadas a $35^{\circ} \mathrm{C}$. Los controles de calidad fueron testeados de la misma manera con Candida parapsilosis ATCC 22019 y Candida krusei ATCC 6258.

Los halos de inhibición fueron medidos en milímetros a las 48 y 72 hs. Se utilizaron los criterios de interpretación de los halos establecidos para Fluconazol (FCZ) y Voriconazol (VOR) por el Clinical and Laboratory Standards Institute en 2006 y al igual que Espinel-Ingroff et al., (20ㅁ), consideramos halos de inhibición frente a Itraconazol $>23$ $\mathrm{mm}$ (sensibles), entre 14 a $22 \mathrm{~mm}$ (sensibles dosis dependientes), y $<13 \mathrm{~mm}$ (resistentes). En el caso de la Anfotericina B (ANB), aún no se han establecido los puntos de corte de CIM o halos de discos para cepas susceptibles o resistentes.

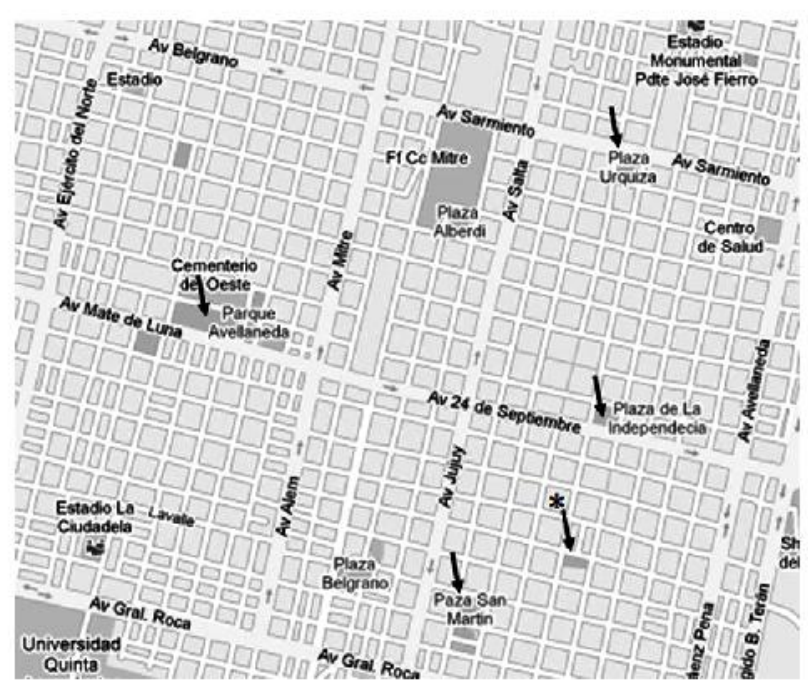

Figura 1. Mapa del radio céntrico de la ciudad de San Miguel de Tucumán indicando los lugares de estudio. (*Plaza Rivadavia)

\section{RESULTADOS}

Del total de 100 muestras de excrementos de paloma, 55 fueron positivas a Cryptococcus. La observación macroscópica en NSA reveló colonias húmedas, brillantes, de color marrón oscuro debido a la acción de la enzima fenol-oxidasa sobre el sustrato. En SGA se observó el desarrollo de colonias lisas, brillantes, húmedas, de consistencia mucoide, de color blanco-crema. A los 4-5 días el color blanco primitivo se fue oscureciendo al tono canela o pardo. El examen microscópico en fresco, con tinta china diluida 1:4, reveló levaduras globosas a ovoides, de 3 a $7 \mu \mathrm{m}$ de diámetro, capsuladas, generalmente con un único brote, sin producción de seudohifas. El resultado de las pruebas morfofisiológicas permitió clasificar a todas las cepas aisladas como $\boldsymbol{C}$. neoformans var. neoformans en base a los siguientes caracteres: ureasa (+), Inositol (+), KNO3 (-), Lactosa (-), Maltosa (+), Sacarosa (+), Galactitol (+), seudomicelio (-), no modifica el color del CGB.

El número de colonias por muestra fue notablemente variable según el microambiente del que fueron aisladas. Cabe destacar que en 11 muestras se obtuvieron cultivos puros a partir del aislamiento ambiental.

C. neoformans var. neoformans se desarrolló en todos los espacios públicos, encontrándose el mayor porcentaje en Plaza Independencia (65\%), y el menor en Plaza Irigoyen (45\%) (Fig. 2).

Todas las cepas testeadas fueron sensibles a FCZ y VOR. Ninguna de las 55 cepas, presentó halos inferiores a $28 \mathrm{~mm}$ frente a ITR, por lo que consideramos que fueron sensibles al mismo. El promedio de la lectura de los halos de inhibición de ANB fue de 21 a $24 \mathrm{~mm}$. 


\section{DISCUSIÓN}

En nuestro estudio, empleamos la detección de los integrantes del género Cryptococcus, mediante la técnica por hisopado, por requerir menor tiempo y trabajo de procesamiento, costos menores y una baja manipulación de material potencialmente infeccioso, diferenciándose de la metodología de otros autores, que emplearon técnicas que permiten un recuento de colonias por gramos del material.

Se determinó la elevada prevalencia, de entre un 45 al 65\%, de Cryptococcus neoformans en las heces de palomas de los principales espacios públicos de la ciudad de San Miguel de Tucumán, donde transitan, cotidianamente, un número muy importante de personas de todas las edades, lo que sugiere un potencial riesgo en salud pública en individuos con compromiso inmune, por la exposición aérea de este microorganismo.

En coincidencia con Chee y Lee (2005) consideramos importante que se efectúen estudios adicionales que cubran otras zonas de la provincia, con mayor número de muestras (especialmente estacionales), a fin de investigar la distribución geográfica y las características de la población del complejo $\boldsymbol{C}$. neoformans presente en las deyecciones de estas aves. Por otro lado, resultaría conveniente que las cepas tipificadas sean comparadas con aislamientos clínicos de nuestra ciudad y sean sometidas a estudios moleculares para poder determinar si estas son las causantes de la infección.

Nuestros resultados confirman los hallazgos de otros autores en el sentido que las excretas de palomas son una importante fuente ambiental y de diseminación de C. neoformans (Caicedo et al., 1996; Curo et al., 2005; Quintero et al., 2005; González-Acuña et al., 2007; Rosario

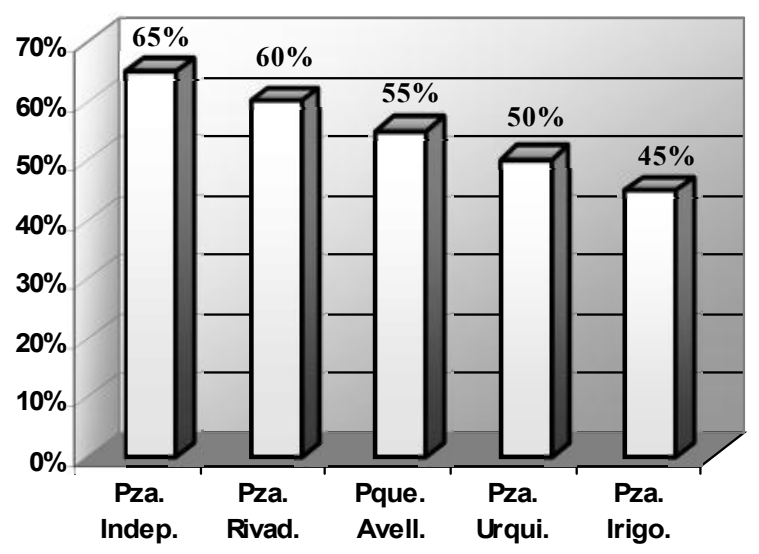

Figura 2. Porcentaje de aislamiento de $C$. neoformans en heces de palomas en 5 espacios públicos de San Miguel de Tucumán, Argentina. 2009. et al., 2008), aunque sólo algunos estudios han encontrado C. neoformans en el cuerpo de estas aves (Rosario et al., 2005). La explicación podría deberse a varios factores tales como: la elevada temperatura interna corporal de las palomas (alrededor $\operatorname{los} 42^{\circ} \mathrm{C}$ ), que imposibilita el desarrollo de $\boldsymbol{C}$. neoformans y a la microbiota bacteriana del contenido intestinal de palomas aparentemente sanas, que inhibe el desarrollo de este patógeno in vitro (Abou-Gabal, 1978). Por otro lado, $\boldsymbol{C}$. neoformans, ha sido recuperado a partir de la superficie corporal de aves incluyendo: plumas, picos, alas y patas, posiblemente por su relación con un hábitat contaminado y enriquecido por las excretas de estas aves (Pal, 1989; Mangiarotti et al., 1993). Además, los excrementos secos representan un sustrato favorable que contienen pocas bacterias y por lo tanto menor competencia, lo que podría explicar su alta frecuencia. (AbouGabal, 1978; Ruiz et al., 1981). Otra hipótesis sugiere que el hongo podría se un geofílico y prosperar en este ambiente particular (Idnurm et al., 2005).

Si se considera que estas aves tienen el hábito de raspar y fragmentar pedazos de madera, ramas y gajos, podríamos suponer que estas costumbres favorecerían la contaminación y diseminación de $\boldsymbol{C}$. neoformans en los árboles que encontramos con presencias positivas en el año 2008 ( Pza. Rivadavia e Irigoyen, Álvarez et al., 2009) lo que sugiere la necesidad de efectuar estudios moleculares a fin confirmar si corresponden a los mismos genotipos.

Existen pocos estudios que evalúan la susceptibilidad antifúngica de cepas ambientales. Entendemos que el presente, es el primer estudio que investiga la actividad in vitro de aislamientos ambientales de $\boldsymbol{C}$. neoformans, recuperados en Tucumán, frente a FCZ, ITR, ANB y VOR. Asumiendo que la sensibilidad de $\boldsymbol{C}$. neoformans a los antifúngicos se encuentra relacionada al área geográfica (Pernan et al., 2006), nuestros datos sobre la susceptibilidad de las cepas ambientales pueden reflejar los patrones para los aislamientos clínicos de nuestra región geográfica.

En concordancia con el estudio realizado en Turquía por Yildiran et al. (2000), con 27 cepas recuperadas de heces de palomas, no encontramos cepas resistentes a FCZ y VOR, mientras Pernan et al., (2006), en una revisión de 2687 aislamientos clínicos de $\boldsymbol{C}$. neoformans, encontraron que sólo el $0,9 \%$ fue resistente a VOR. Si bien utilizamos el método de difusión en placa de agar MHM, en lugar de aplicar la técnica gold standard (método de macro o microdilución), sustentamos nuestro resultado en los estudios de Espinel-Ingroff et al., (2007), quienes obtuvieron una buena correlación con las tabletas de NeoSensitab a los mismos antifúngicos. Asimismo, en el estudio de Pfaller et al. (2004), esta técnica permitió la identificación de cepas de $\boldsymbol{C}$. neoformans sensibles dosis dependiente al FCZ.

Los puntos de corte para la concentración inhibitoria 
mínima (CIM) para ITR, han sido establecidos, pero aún no hay guías disponibles para la interpretación de las pruebas con discos. Por ello, aplicamos los mismos criterios que Espinel-Ingroff et al., (2007), hallando en todas las cepas diámetros mayores a los $5 \mathrm{~mm}$ del punto de corte considerados como sensibles. Datos similares fueron obtenidos en España por Carrillo-Muñoz et al., (1997), donde todos los aislamientos clínicos de $\boldsymbol{C}$. neoformans fueron sensibles a ITR y también a FCZ e Imidazoles.

A pesar de los problemas metodológicos asociados a ANB, y que Espinel-Ingroff et al., (2007) no pudieron identificar cepas resistentes a ANB yque además tuvieron baja correlación al usar Neo-Sensitab en comparación con el análisis de microdilución (CLSI: documento M27-A2) y de difusión en disco (CLSI: documento M44-A); consideramos necesario efectuar esta prueba, ya que el tratamiento de predilección frente a la meningitis por $\boldsymbol{C}$. neoformans es ANB. Los resultados logrados frente a ANB, sugieren que se trataría de cepas sensibles según los puntos de corte sugeridos por el fabricante $(>15 \mathrm{~mm})$, pero pensamos que deberían ser comparados en un futuro cuando se disponga de zonas de diámetro de referencia para ANB.

Por otra parte, el presente estudio es de interés si se considera el marcado incremento de la incidencia de esta micosis asociada con el crecimiento poblacional de pacientes inmunodeprimidos y pacientes infectados con VIH, debido que en la provincia de Tucumán se registraron más de 700 diagnósticos en el período 1987-2009 (UCA, 2010). De aquí la importancia de métodos de control que eviten la acumulación de las excretas de palomas en la ciudad, mediante la limpieza periódica de las mismas, especialmente en zonas aledañas a los centros hospitalarios.

Igualmente, juzgamos que es necesario profundizar estos estudios y establecer mecanismos de vigilancia epidemiológica, ya que los resultados revelan un alto grado de positividad frente a un hongo patógeno oportunista.

\section{REFERENCIAS}

Abou-Gabal, M. \& Atia, M. (1978). Study of the role of pigeons in the dissemination of Cryptococcus neoformans in nature. Sabouraudia 16:63-68

Álvarez, C.; Salim, R. \& Runco, R. (2009). Aislamiento de Cryptococcus neoformans desde madera en descomposición de Jacaranda mimosifolia y Enterolobium contortisiliquum. Boletín Micológico 24:15-20

Bava, A.J. \& Negroni, R. (1986). Estudio epidemiológico sobre Cryptococcosis en San Pedro Provincia de Buenos Aires, Argentina. Rev. Argentina de Micología 9(3):12-6
Bernardo, F.; Martins, M. \& Lígia, M. (2001). Fontes urbanas de Cryptococcus spp. Lisboa. Rev Port Ciênc Vet; 96 (539): 15760

Caicedo, L.; Alvarez, M.; Llanos, C.; Molina, D. (1996). Cryptococcus neoformans en excreta de palomas del perímetro urbano de Cali. Colombia Med. 27:106-9

Carrillo-Muñoz, A.J.; Tur, C.; Estivill, D.; Montsant, L.; Carceller, A.; Hernandez, J.M.; Torres-Rodriguez, J.M. (1997). Resistencia in vitro al fluconazol e itraconazol en aislamientos clínicos de Candida spp. y Cryptococcus neoformans Rev. Iberoam Micol. 14:50-54.

Casadevall, A. \& Perfect, J.R. (1998). Cryptococcus neoformans. Washington D.C., ASMPress

Cermeño, J.; Hernández, I.; Cabello, A.; Caraballo, Y.; Orellán, A.; Padrón, J. (2002). Aislamiento de Cryptococcus neoformans e Histoplasma capsulatum en excreta de palomas, en Ciudad de Bolívar, Venezuela. En: Libro de Resúmenes del IV Congreso Latino Americano de Micología. Veracruz: Sociedad Latinoamericana de Micología p. 435

Chee, H.Y. \& Lee, K.B. (2005). Isolation of Cryptococcus neoformans var. grubii (serotype A) from Pigeon Droppings in Seoul, Korea. Journal of Microbiology. 43:469-472

Clinical and Laboratory Standards Institute. (2006). Zone diameter interpretive standards and corresponding minimal inhibitory concentration (MIC) interpretive breakpoints. SupplementM44-S1. Clinical and Laboratory Standards Institute, Wayne, PA.

Colom Valiente, M.F.; Alberdi, M.; Meseguer, I.; Torres, J. (1997). Aislamiento de Cryptococcus neoformans en muestras de medio ambiente de Alicante. Rev Iberoam Micol 14:63-64

Curo, M.I.; Salinas, M.F. \& Casquero, J. C. (2005). Cryptococcus neoformans en excretas de palomas, suelo y aire de los palomares del perímetro urbano de Ica. Rev. Peru. Med. Exp. Salud Publica 22: 262-266

Ellis, D.H. \& Pfeiffer, T.J. (1990). Natural habitat of Cryptococcus neoformans var. gattii. J. Clin. Microbiol. 28:1642-1644

Emmons, C.W. (1955). Saprophytic sources of Cryptococcus neoformans associated with the pigeon (Columba livia). Am. J. Hyg. 62:227-232

Emmons, C.W. (1960). Prevalence of Cryptococcus neoformans in pigeon habitats. Publ Health Rep 75:362-364

Emmons, C.W. (1995). Saprophytic sources of Cryptococcus neoformans associated with the pigeon (Columba livia). Am. J. Hyg. 62:227-232

Espinel-Ingroff A.; Canton, E.; Gibbs, D. \& Wang, A. (2007). Correlation of Neo-Sensitabs Tablet Diffusion Assay Results on Three Different Agar Media with CLSI Broth Microdilution M27A2 and Disk Diffusion M44-A Results for Testing Susceptibilities of Candida spp. and Cryptococcus neoformans to Amphotericin B, Caspofungin, Fluconazole, Itraconazole, and Voriconazole. Journal of Clinical Microbiology. 45:858-864 
González-Acuña, D.; Silva, G.F.; Moreno, S.L.; Cerda, L.F.; Donoso, E.S.; Cabello, C.J.; y López, M.J. (2007). Detección de algunos agentes zoonóticos en la paloma doméstica (Columba livia) en la ciudad de Chillán, Chile. Rev. Chil. Infect. 24 :194-198

Granados, D.P. \& Castañeda, E. (2005). Isolation and characterization of Cryptococcus neoformans varieties recovered from natural sources in Bogota, Colombia, and study of ecological conditions in the area. Microb. Ecol. 49:282-90

Heitman, J. \& Lin, X. (2006) The biology of the Cryptococcus neoformans species complex. Rev Microbiol. 60:69-105

Hoang, L.M.; Maguire, J.A.; Doyle, P.; Fyfe, M.; Roscoe, D.L.. (2004). Cryptococcus neoformans infections at Vancouver Hospital and Health Sciences Centre (1997-2002): epidemiology, microbiology and histopathology. J. Med. Microbiol. 53:935-940

Hsu, M.M.; Chang, J.C.; Yokoyama, K.; Nishimura, K.; Miyaji, M. (1994). Serotypes and mating types of clinical strains of Cryptococcus neoformans isolated in Taiwan. Mycopathologia 125:77-8

Idnurm, A.; Bahn, Y.S. ; Nielsen, K.; Lin, X.; Fraser, J.A.; Heitman, J. (2005). Deciphering the model pathogenic fungus Cryptococcus neoformans. Nat. Rev. Microbiol. 3:753-764

Kielstein, P. \& Bocklish, H. (2000a). Evidence of Cryptococcus neoformans in domestic and sports pigeons in Thuringia, Germany. Mycoses 43, 23-28

Kielstein, P.; Hotzel, H.; Schmalreck, A.; Khaschabi, D.; Glawischnig, W. (2000b). Occurrence of Cryptococcus spp. in excreta of pigeons and pet birds. Mycoses 43:7-15

Kwon-Chung, K.J.; Boekhout, T.; Fell, J.W. \& Diaz, M. (2002) Proposal to converse the name Cryptococcus gattii against $\boldsymbol{C}$. hondurianus and C. ballisporus (Basidiomycota, Hymenomycetes, Tremellomycetidae). Taxon 51:804-806

Kwon-Chung, K.J. \& Varma, A. (2006). Do major species concept support one, two or more species within Cryptococcus neoformans. FEMS Yeast Res. 6:574 - 587

Lazera, M.S.; Pires, F.; Camillo-Coura, L.; Nishikawa, M.; Bezerra, C.F.; Trilles, L. (1996). Natural habitat of Cryptococcus neoformans var. neoformans in decaying wood forming hollows in living trees. J. Med. Vet. Mycol. 34:127-131

Li, A.; Nishmura, K.; Taguchi, H.; Takana, R.; Wu, S.; Miyaji, M. (1993). The isolation of Cryptococcus neoformans from pigeon droppings and serotyping of naturally and clinically sources of isolates in China. Mycopathologia 124:1-5

López-Martínez, R. \& Castañón-Olivares, L.R. (1995). Isolation of Cryptococcus neoformans var. neoformans from bird droppings, fruits and vegetables in Mexico City. Mycopathologia $129: 25-28$

Mangiarotti, A.M.; Caretta, G.; De Luca, Carla. \& Piontelli, E. (1993). Hongos aislados del plumaje y excrementos de gallinas en una industria avícola de Monferrato (Pavia-Italia). Bol. Micol. 8: 91-98

Mattsson, R.; Haemig, P.D. \& Olsen, B. (1999). Feral pigeons as carriers of Cryptococcus laurentii, Cryptococcus uniguttulatus and Debaryomyces hansenii. Medical Mycology 37:367-370
Pal, M. \& Mehrotra, B.S. (1984). Studies on the isolation of Cryptococcus neoformans from fruits and vegetables. Mykosen 28:200-205

Pal, M. (1989). Cryptococcus neoformans var. neoformans and munia birds. Mycoses 32:250-52

Passoni, L.F.C. (1999) Wood, animals and human beings as reservoirs for human Cryptococcus neoformans infection. Rev. Iberoam Micol. 16:77-81.

Pernan, J.; Canton, E.; Calabuig, E.; Bosch, M.; Valentín, A.; Viudes, A.; Gobernado, M. (2006). Actividad in vitro del voriconazol frente a levaduras y algas con los nuevos puntos de corte del patrón de resistencia. Rev. Esp. Quimioterap. 19:21-33

Pfaller, M.A.; Messer, S.A.; Boyken, L.; Rice, C.; Tendolkar, S.; Hollis, R. J.; Diekema, D. J. (2004). Evaluation of NCCLSM44-P disk diffusion method for determining susceptibilities of 276 clinical isolates of Cryptococcus neoformans to ûuconazole. J. Clin. Microbiol. 42:380-383

Pollock, C. (2003). Fungal diseases of columbiformes and anseriformes. Veterinary Clin. North. Am Exot Anim Pract; 6:351-361

Quicaño, L.; Romero, S.; Zurita, S. \& Casquero, J. (1999). Aislamiento de Cryptococcus neoformans en heces, suelo y aire de viviendas con palomas domésticas «Columbia livia» - Ayacucho. En: Libro de Resúmenes del III Congreso Latinoamericano de Micología. Venezuela: Sociedad Latinoamericana de Micología; p. 101.

Quintero, E.; Castañeda, E. \& Ruiz, A. (2005). Distribución ambiental de Cryptococcus neoformans en el departamento de Cundinamarca-Colombia. Rev. Iberoam. Micol. 22:93-98

Rivas, F.; De Martin, M.C. \& Rojas, V. (1999). Primer aislamiento de Cryptococcus neoformans a partir de suelos en Panamá. Rev. Med. Panama. 24:4-6

Rosario, I.; Hermoso de Mendoza, M.; Deniz, S.; Soro, G.; Alamo, I.; Acosta, B. (2005). Isolation of Cryptococcus species including $\boldsymbol{C}$. neoformans from cloaca of pigeons. Mycoses 48:42124

Rosario, I.; Acosta, B. \& Colom, F. (2008). La paloma y otras aves como reservorio de Cryptococcus spp. Rev. Iberoam. Micol. 25:S13-S18

Rubinstein, H.; Marticorena, B. \& Masih, D. (1989). Isolation of human fungi from soil and identification of two endemic areas of Cryptococcus neoformans and Coccidioides immitis. Rev. Inst. Med. Trop. São Paulo 31:1-6

Ruiz, A.; Fromtling, R.A. \& Bulmer, G.S. (1981). Distribution of Cryptococcus neoformans in a natural site. Infect. Immun. $31: 560-63$

Sammann, S.; Diaz, L.; Salamanca, F. \& Prado, V. (1994). Cryptococcus neoformans (San Felice Vuillemin) en aves confinadas y recintos hospitalarios de la región Metropolitana (Chile). Boletín Mycol. 9 :65-72

Silva, M. \& Luiza, P. (1963). Isolamento de Cryptococcus neoformans de excrementos e ninhos de pombos (Columba livia) em Salvador, Bahia (Brasil). Rev Inst Med Trop São Paulo 5:9-11 
Staib, F. \& Blisse, A. (1982). Bird manure ûltrate agar for the formation of the perfect state of Cryptococcus neoformans, Filobasidiella neoformans. A comparative study of the agars prepared from pigeon and canary manure. Zentralbl. Bakteriol. Mikrobiol. Hyg. A 251:554-62

Staib, F. (1987). Cryptococcosis in AIDS-Mycological diagnostic and epidemiological observations. AIFO. 2:363-382

Tampieri, M.P. (2006). Mycetes and urban areas. Parassitologia 48:121-124

Taubenberger, J. K. \& Morens, D. M. (2006). 1918 inûuenza: the mother of all pandemics. Emerg. Infect. Dis. 12:15-22

U.C.A. Unidad Coordinadora y Ejecutora Provincial VIH/SIDA y ETS. SIPROSA. Tucumán. (2010). http://www.msptucuman.gov.ar/ archivos/pdf/SALA_7_junio_2010_web.pdf
Vanni, C.; Sarsotti, P.V.; Ruatta, J.; García, G.; Gutierrez, C.; Cabagna, M. (1998). Ecología del Cryptococcus neoformans en Santa Fe. Revista FABICIB $2: 25-30$

Yehia, A. : Mahmoud, G. (1999). First environmental isolation of Cryptococcus neoformans var. neoformans and var. gattii from the Gharbia Governorate, Egypt. Mycopathologia 148:83-86

Yimtubezenash, W.A.; Jemaneh, L. \& Abate, K. (2001). Isolation and characterization of Cryptococcus neoformans from environmental sources in Ethiopia. Ethiop.J.Health Dev. 15:45-50

Yildiran S.T.; Sarachi M.A.; Fothergill A.W. \& Rinaldi M.G. (2000). In vitro suceptibility of environmental Cryptococcus neoformans var. neoformans isolates form Turkey to six antifungal agents, including SCH56592 and Voriconazole. Journal Clin. Microb. Infect. Dis. 19:317-319 